\title{
Osteonecrosis of the femoral head in people living with HIV: anatomopathological description and p24 antigen test
}

This article was published in the following Dove Press journal: HIVIAIDS - Research and Palliative Care

\author{
Ana Lucia L Munhoz Lima' \\ Priscila Rosalba Oliveira' \\ Vladimir C Carvalho' \\ Alexandre Leme \\ Godoy-Santos' \\ Leandro Ejnisman' \\ Claudia R Oliveira' \\ David E Uip ${ }^{2}$ \\ Maria Irma S Duarte \\ Instituto de Ortopedia e \\ Traumatologia, Hospital das Clínicas \\ HCFMUSP, Universidade de São \\ Paulo, São Paulo, Brazil; ${ }^{2}$ Faculdade \\ de Medicina da Fundacao do ABC, \\ São Paulo, Brazil; ${ }^{3}$ Departamento \\ de Patologia, Faculdade de Medicina \\ FMUSP, Universidade de São Paulo, \\ Sao Paulo, Brazil
}

Objective: To examine the presence of HIV in bone tissue of people living with HIV (PLWHIV) with osteonecrosis of femoral head and describe clinical and anatomopathological findings.

Design: This is a case series which included 44 PLWHIV with osteonecrosis of femoral head who underwent total hip arthroplasty.

Methods: Clinical data were obtained through analysis of the patients' medical records. Bone tissue obtained during total hip arthroplasty was retrieved and sent for conventional and immunohistochemical analysis. Monoclonal antibodies were used to mark the p24 (HIV), CD31 (vascular endothelial cells), CD68 (macrophages), and $\mathrm{D}_{2} 40$ (cells of the lymphatic endothelium) antigens. Results: Dyslipidemia was found in $48 \%$ of the patients and lipodystrophy in $31 \%$. Histological analysis showed similar characteristics for the entire sample. Degeneration of joint cartilage was visualized with the presence of fissures and fibrillations, as well as subchondral sclerosis and necrosis of the subchondral cancellous bone tissue. Lymphoplasmocytic inflammatory reaction was observed, with the presence of macrophages containing a foamy, vacuolated cytoplasm, as well as the presence of ceroid pigment and occasional granulation tissue. The reaction with the monoclonal anti-p24 antibody was negative in the samples from all 44 PLWHIV undergoing hip arthroplasty. Reactions with the anti-CD31 and anti-D 40 antibodies were negative. Staining with CD68 antibody confirmed that the cells visualized with foamy, vacuolated cytoplasm were macrophages.

Conclusion: p24 HIV antigen was not detected in the bone tissue of PLWHIV and osteonecrosis. The most frequent anatomopathological findings were extensive necrosis of bone tissue, large vacuoles filled with fat cells, inflammatory lymphoplasmocytic reaction with macrophages containing vacuolated cytoplasm, and the presence of ceroid pigment.

Keywords: osteonecrosis, AIDS, HIV core protein p24, immunohistochemistry

\section{Introduction}

People living with HIV (PLWHIV) develop a chronic inflammatory syndrome and persistent immune deregulation, which interfere with various metabolic responses, including the metabolism of bone tissue through the action of cytokines and other immune factors involved in the maturation of osteoblasts and reabsorption of osteoclasts. ${ }^{1}$ In addition to the direct action of pro-inflammatory cytokines in bone tissue, there are marked changes in neuroendocrine-immune system regulation due, in part, to changes in parathyroid hormone (PTH) and calcitonin. These changes in PTH levels are particularly important because they regulate the production of pro-inflammatory cytokines such as IL- 6 and TNF- $\alpha$, which stimulate osteoclastogenesis and bone resorption and, at the nuclear factor kappa-B receptor binding site, encourage the formation
Correspondence: Priscila R Oliveira Rua Doutor Ovidio Pires de Campos, 333, sala 3IIA, 05403-010 São Paulo, Brazil

Tel +55 II 266 I 6900

Email ana.munhoz@hc.fm.usp.br 
of osteoclasts, thereby increasing bone turnover. ${ }^{2,3}$ Among PLWHIV who have not yet been treated, an uncoupling is seen between bone formation and resorption, due to the action of the virus itself, or rather, the immune-mediated effects of HIV on the skeleton, generating varying degrees of osteopenia and osteoporosis.

Osteonecrosis of the femoral head is closely related to the presence of HIV and highly active antiretroviral therapy (HAART). This is a prevalent disease, with clinical significance and poor prognosis in this population. The occurrence of osteonecrosis has been reported in this group of patients since 1990, with an incidence higher than that of the general population. ${ }^{4,5}$ The annual incidence of symptomatic osteonecrosis in the general population is estimated to range from $0.010 \%$ to $0.135 \%{ }^{6}$ In magnetic resonance imaging of PLWHIV without femoroacetabular joint pain, a prevalence of $4.4 \%$ of hip osteonecrosis, with bilaterality ranging from $35 \%$ to $80 \%$, is observed. Furthermore, the reported incidence of symptomatic disease is approximately a 100 times higher than in the general population. ${ }^{6,7}$ Whereas the osteonecrosis level in the general population has stabilized in recent years, higher rates of disease have been observed among PLWHIV.

The etiology of osteonecrosis remains unclear, although the following risk factors have been identified for the general population: use of systemic corticosteroids, alcohol abuse, hyperlipidemia, sickle cell anemia, coagulopathy, Gaucher's disease, systemic lupus erythematosus, rheumatoid arthritis, hyperuricemia and gout, radiotherapy, obesity, pancreatitis, sequelae of fractures, chemotherapy, vasculitis, and smoking. In addition to these factors, in patients with HIV/AIDS, risk factors for the development of osteonecrosis include dyslipidemia, use of megestrol acetate and anabolic steroids, and testosterone replacement, as well as vasculitis due to the presence of anticardiolipin antibodies and protein S deficiency, which predispose patients to intraosseous thrombosis. In addition, the antiretroviral treatment itself may be related to the development of osteonecrosis. However, based on the available data, neither the actions of the virus alone nor the antiretroviral treatment constitute independent risk factors for osteonecrosis. ${ }^{4-7}$

The treatment of osteonecrosis is difficult, and in this population group in particular, despite therapeutic measures, disease progression is usually rapid. The treatment options are indicated by the stage of the disease and the clinical presentation. The surgery most often used for the treatment of advanced osteonecrosis of the femoral head is total hip arthroplasty (THA).
The objectives of this study were to examine the presence of HIV in the bone tissue of PLWHIV with osteonecrosis undergoing THA and to describe the anatomopathological findings, comparing with samples from HIV-negative patients as negative control. The secondary objective was to describe the clinical characteristics of the population studied.

\section{Methods}

This study was reviewed and approved by the Ethics Commission for Analysis of Research Projects - CAPPesq (Comissão de Ética para Análise de Projetos de Pesquisa) of Hospital das Clínicas Medical School of the Universidade de São Paulo (protocol number 606/08). This study was retrospective and included patients who had already been operated. Therefore, consent to review their medical records was not required by CAPPesq. Patient data were extracted from medical records to a standardized form and then to an electronic chart. The forms had identified data and were used only by the research team. We used only deidentified data for the statistical analysis and paper.

This case series included 44 PLWHIV with stage 3 and stage 4 osteonecrosis of the femoral head, graded according to the classification system of Ficat and Arlet. The included patients had either unilateral or bilateral osteonecrosis and underwent THA for correction of the deformity. All patients received confirmation of osteonecrosis by magnetic resonance imaging of the hip. Seven patients had bilateral involvement and were submitted to bilateral arthroplasty. A total of 51 hip arthroplasties were performed in 44 patients.

Data on the following variables were obtained through analysis of the patients' medical records: age, gender, unilateral or bilateral involvement of osteonecrosis, time of HIV infection to arthroplasty in years, laterality, history of previous surgery on the affected topography, CD4 lymphocyte counts at the time of surgery, HIV viral load at the time of surgery, history of alcoholism or smoking, previous diagnosis (dyslipidemia, lipodystrophy, diabetes mellitus, and osteoporosis), antiretroviral drugs in use at the time of surgery, and time of exposure prior to each of the classes of antiretrovirals (nucleoside reverse transcriptase inhibitors [NRTIs], non-nucleoside reverse transcriptase inhibitors [NNRTIs], protease inhibitors [IPs], and integrase inhibitors).

All THAs were performed using standard techniques. ${ }^{8}$ All implants were uncemented, and the bearing surface was ceramic on ceramic. After femoral neck osteotomy, the femoral head was retrieved and sent for histological analysis. 
Conventional histological analysis was performed as follows. The femoral heads were cut into sagittal slices with a thickness of $4 \mu \mathrm{m}$ and then fixed in a $10 \%$ formalin solution $\left(\mathrm{H}_{2} \mathrm{CO}\right)$ for 24 hours. Next, the bone component was decalcified in $7.5 \%$ nitric acid $\left(\mathrm{HNO}_{3}\right)$ for $\sim 48$ hours. After histotechnical processing, the material was stained with H\&E. Five to eight sections were prepared for representation.

For the immunohistochemical analysis, monoclonal antibodies were used to mark the p24 (HIV), CD31 (vascular endothelial cells), CD68 (macrophages), and $\mathrm{D}_{2} 40$ (cells of the lymphatic endothelium) antigens. For staining, the material was incubated with biotinylated universal secondary antibody (anti-mouse, anti-rabbit, and anti-goat) from the LSAB peroxidase kit (LSAB + system-HRP, code K0690; DakoCytomation, Glostrup, Denmark) for the reaction of CD68, the Polymer System of the Novolink kits (Max Polymer Detection System, REF: RE7280-K; Leica) for the reactions of $\mathrm{p} 24$ and $\mathrm{CD} 31$, and the Advance kit (REF: K4068; DakoCytomation) for the reaction of $\mathrm{D}_{2} 40$. The following reagents were also used: streptavidin peroxidase from the LSAB peroxidase kit (LSAB + system-HRP, code K0690; DakoCytomation), HRP enzyme (Advance), and Polymer (Novolink).

To validate the results obtained in the immunohistochemical reaction to the $\mathrm{p} 24$ antigen, samples of lymph node tissue were obtained from HIV-infected patients, a known reagent for this antigen, and used as positive controls. In addition, samples of bone tissue from 10 patients diagnosed with osteonecrosis of the femoral head, known to be HIV-negative and negative for $\mathrm{p} 24$, were obtained during THA and used as negative controls.

The quantitative clinical variables evaluated were described by group, using summary measurements (mean, $\mathrm{SD}$, median, minimum, and maximum), and the qualitative variables were described using relative and absolute frequencies. ${ }^{9}$ For the histological and immunohistochemical variables, descriptive analyses were carried out. The data were inputted using Microsoft Office Excel 2010 (Microsoft Inc, Redmond, WA, USA) and analyzed using SPSS 20.0 (IBM Corporation, Armonk, NY, USA).

\section{Results}

Forty-four patients, predominantly males, were included in the study. The patients' ages ranged from 31 to 67 years, with an average of 46 years. The time of HIV infection ranged from 4 to 24 years, with an average of 12 years. The CD4 lymphocyte counts in the peripheral blood of the patients at the time of surgery ranged from 55 to 1,268 cells $/ \mathrm{mm}^{3}$, with a mean count of $\sim 525$ cells $/ \mathrm{mm}^{3}$. The vast majority of the samples, $\sim 88 \%$, presented with an undetectable HIV viral load at the time of surgery. Of the five patients (11.6\%) with a detectable viral load, $60-550,000$ viral copies $/ \mathrm{mm}^{3}$ were present in the peripheral blood. All the patients included in the study reported receiving HAART at the time of surgery. Detailed data on antiretroviral use were available for 30 patients. Of these, the length of time using these medications ranged from 4 to 21 years, with an average time of use of 10 years. The number of antiretroviral regimens prior to the current treatment ranged from 1 to 9 , with an average of four treatment regimens prior to the current treatment. In relation to the metabolic changes commonly associated with HIV infection and antiretroviral therapy, the prevalence of dyslipidemia was $\sim 48.0 \%$, lipodystrophy was $31.0 \%$, and hyperglycemia was $13.6 \%$. There was only one confirmed case of a patient with osteoporosis. The prevalence of previous alcoholism was $50.0 \%$, and that of smoking, whether previous or current, was $77.2 \%$. The majority of the 44 patients studied had bilateral involvement of the hips with osteonecrosis $(70.5 \%)$. The vast majority of the patients $(93.2 \%)$ had no history of a surgical procedure in the same arthroplasty topography. In relation to laterality, the majority of cases were operated to treat the left hip (56.8\%). Table 1 shows the main clinical and demographic data of the studied sample.

In relation to the classes of antiretroviral drugs to which these patients were exposed, there was a predominance of NRTIs, with a history of use (previous or current) in $100.0 \%$ of the patients and an average time of use of $\sim 10$ years. There was a history of use of NNRTIs in $\sim 83.0 \%$ of patients and an average time of use of $\sim 4$ years. IPs, in turn, were used at some time during the treatment by $\sim 67.0 \%$ of the patients, with an average time of use of $\sim 5$ years (Table 2 ).

Histological analysis of the femoral heads of the 44 patients showed similar characteristics for the entire sample, as shown in Figure 1. Degeneration of the joint cartilage was visualized, with the presence of fissures and fibrillations, compatible with osteoarthrosis, as well as subchondral sclerosis and necrosis of the subchondral cancellous bone tissue. The bone matrix maintained eosinophilic staining with empty gaps. The bone marrow showed areas of adiponecrosis and granular eosinophilic amorphous material with debris and cell remains. A lymphoplasmocytic inflammatory reaction was observed, with the presence of macrophages containing a foamy, vacuolated cytoplasm as well as the presence of ceroid pigment and occasional granulation tissue. The 
Table I Main demographic characteristics and those related to HIV infection and ART

\begin{tabular}{|c|c|}
\hline Variables & Characteristics $(n=44)$ \\
\hline \multirow[t]{2}{*}{ Gender } & Male - $37(84.1 \%)$ \\
\hline & Female - 7 (I5.9\%) \\
\hline Time of HIV infection in years (average) & $11.9(4-24)$ \\
\hline $\begin{array}{l}\text { CD4 lymphocyte count } / \mathrm{mm}^{3} \text { of } \\
\text { peripheral blood (average) }\end{array}$ & $534.7(55-1,238)$ \\
\hline \multirow[t]{2}{*}{ HIV viral load } & Undetectable - 39 (88.4\%) \\
\hline & Detectable - 5 (I I.6\%)* \\
\hline $\begin{array}{l}\text { Time of exposure to ART in years } \\
\text { (average) }\end{array}$ & $|0.|(4-2 \mid)^{* *}$ \\
\hline $\begin{array}{l}\text { Number of ART regimens used } \\
\text { (average) }\end{array}$ & $4(I-9)^{* *}$ \\
\hline \multirow[t]{4}{*}{ Metabolic changes } & Dyslipidemia - 21 (47.7\%) \\
\hline & Lipodystrophy - 13 (30.9\%) \\
\hline & Hyperglycemia - 6 (13.6\%) \\
\hline & Osteoporosis - I (2.2\%) \\
\hline Alcoholism & $22(50.0 \%)$ \\
\hline Smoking & $34(77.2 \%)$ \\
\hline Steroids use & I $(2.2 \%)$ \\
\hline \multirow[t]{2}{*}{ Osteonecrosis } & Bilateral - 3I (70.5\%) \\
\hline & Unilateral - I3 (29.5\%) \\
\hline
\end{tabular}

Notes: *Variation between 60 and 550,000 copies $/ \mathrm{mm}^{3}$ average of 132,518 copies/ $\mathrm{mm}^{3}$. **Information available for 30 of the 44 patients.

Abbreviation: ART, antiretroviral therapy.

Table 2 Information about the use of antiretrovirals in the study population

\begin{tabular}{lll}
\hline Class of ARVs & $\begin{array}{l}\text { Patients with previous } \\
\text { or current use }(\mathbf{n}=\mathbf{3 0})\end{array}$ & $\begin{array}{l}\text { Time of use in years } \\
\text { (average) }\end{array}$ \\
\hline NRTIs & $30(100.0 \%)$ & $10.1(4-2 I)$ \\
NNRTIs & $25(83.3 \%)$ & $4.3(I-I I)$ \\
IPs & $20(66.7 \%)$ & $5.2(I-I 4)$ \\
\hline
\end{tabular}

Abbreviations: ARVs, antiretrovirals; NRTIs, nucleoside reverse transcriptase inhibitors; NNRTls, nonnucleoside reverse transcriptase inhibitors; IPs, protease inhibitors.

macrophage counts per high-magnification field $(40 \times 10)$ ranged from 16 to 40 cells.

The reaction with the monoclonal anti-p24 antibody was strongly positive for lymph node samples from patients known to be living with HIV (positive control), demonstrating the specificity of this reaction. In contrast, the reaction was negative for bone tissue from the 10 patients known to be HIV negative. The reaction was also negative in the samples from all 44 PLWHIV undergoing hip arthroplasty (Figure 2).

For these patients, reactions with the monoclonal antiCD31, anti-CD68, and anti- $\mathrm{D}_{2} 40$ antibodies were also performed to confirm the findings observed in conventional histopathology. The reactions with the anti-CD31 and anti- $\mathrm{D}_{2} 40$ antibodies were negative, showing that the gaps visualized were not related to the presence of blood vessels or lymphatic vessels. Staining with the CD68 antibody confirmed that the cells visualized with foamy, vacuolated cytoplasm were macrophages (Figure 3).

\section{Discussion}

Osteonecrosis of the femoral head typically affects young and active individuals, leading to progressive loss of function. Although its incidence has stabilized in the general population in recent years, it has been increasing among PLWHIV. ${ }^{10}$ Indeed, the incidence of osteonecrosis in this population is 40 times higher than in the general population, with faster progression between stages of the disease, more frequent bilaterality, and more intense symptoms, especially in relation to pain. ${ }^{11}$ Although no etiological agent or pathophysiological mechanism has been clearly identified, some relevant theories have been proposed in regard to the genesis of the process, such as changes in intravascular blood flow, direct cell toxicity, and more recently, impaired mesenchymal cell differentiation. Some authors defend the influence of alcohol abuse and steroid use in the development of osteonecrosis, having observed the profound effect of these two aggressors on the differentiation of bone marrow stromal cells and the blood supply, leading to decreased bone turnover and oxygenation. However, there is no consistent explanation for the absence of osteonecrosis in PLWHIV who are alcohol abusers, some of whom also show the prolonged use of steroids. This observation leads us to speculate that predisposing genetic conditions may be responsible for the development of osteonecrosis, such as specific polymorphisms in the genes encoding nitric oxide synthase or type II collagen..$^{10,12,13}$ It is important to emphasize that the majority of patients present a combination of the various predisposing conditions and risk factors traditionally associated with osteonecrosis. ${ }^{10}$ Moreover, the risk factors associated with osteonecrosis in patients living with HIV can include smoking, alcohol abuse, hyperlipidemia, lipodystrophy, prolonged use of steroids, the presence of anti-phospholipid and anti-cardiolipin antibodies, protein S deficiency, and the duration of HIV infection and antiretroviral treatment. The possibility of direct viral involvement in the bone tissue when considering the etiology of vascular alterations that initiate the process of necrosis has not yet been described.

In the largest case series of PLWHIV/AIDS and osteonecrosis, the epidemiological profile described was quite similar to that found in our group of patients. ${ }^{14-16}$ The present case series included 44 patients, mostly men, with a mean age of 46 years, an average time of HIV infection of 12 years, a mean CD4 lymphocyte count of 525 cells $/ \mathrm{mm}^{3}$, and the 


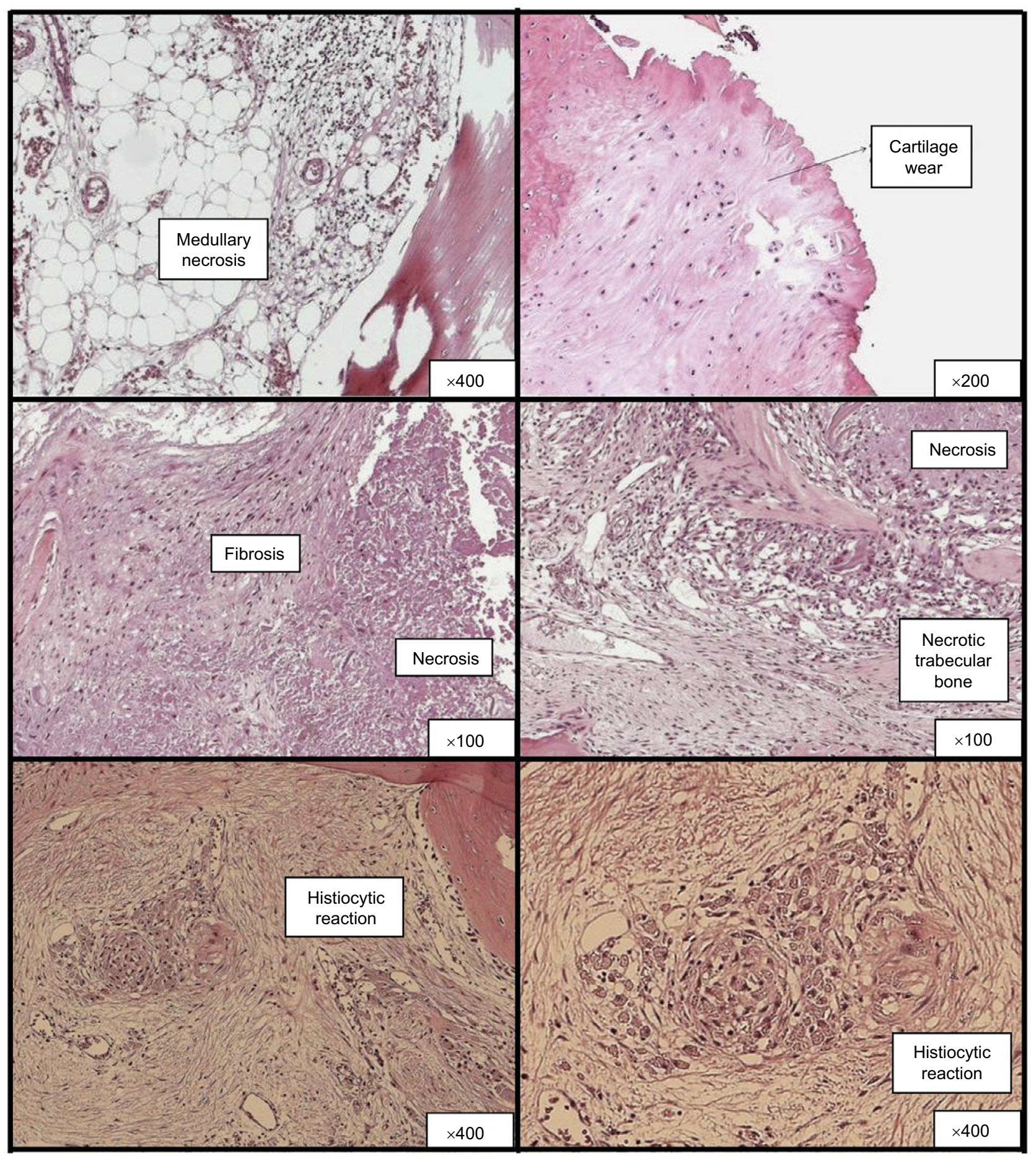

Figure I Panel showing frequent changes in histological sections of femoral bone tissue of patients living with HIV and undergoing THA. Abbreviation: THA, total hip arthroplasty.

majority with undetectable HIV viral loads. The average time of use of antiretroviral drugs was 10 years, with the use of three major classes of drugs for more than 4 years, separately or combined. This information clearly shows that the studied population consisted mostly of patients taking multiple antiretroviral drug regimens over the course of disease evolution. Therefore, this was conceptually considered a multi-experienced population.

Of the 44 cases analyzed in our study, we identified changes in serum lipids, that is, dyslipidemia in $48 \%$ and lipodystrophy in $31 \%$ of the patients. This finding corroborates those of previous published studies, which indicate that 


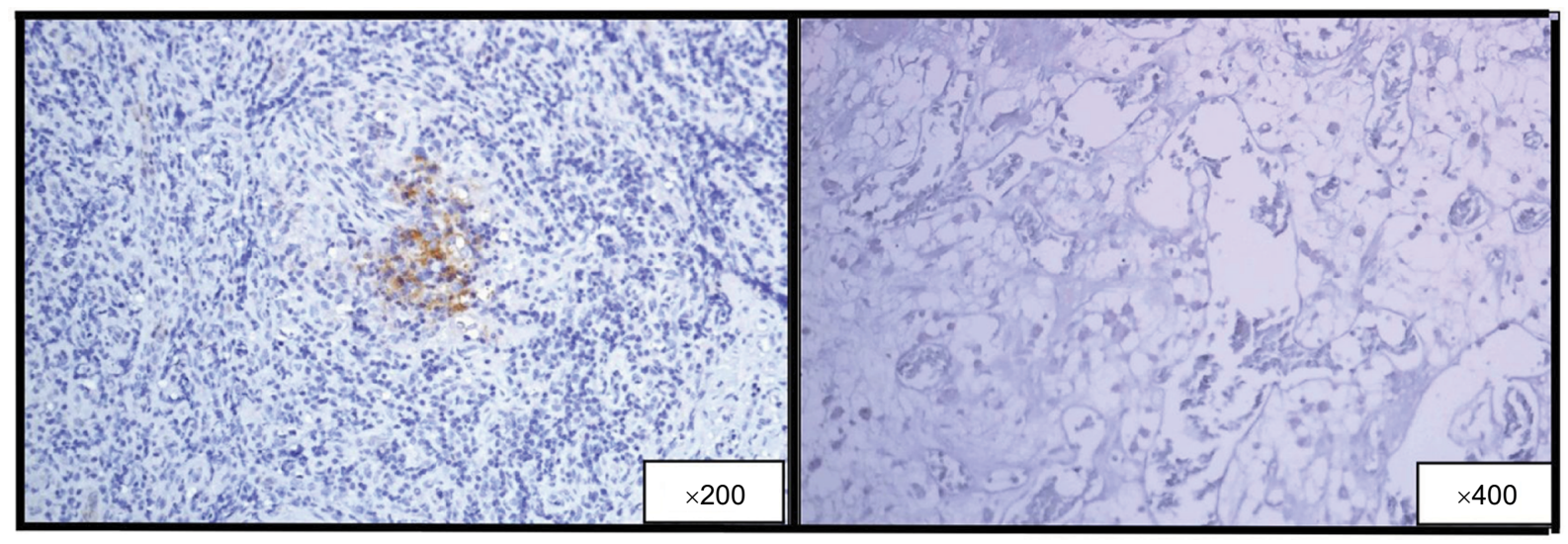

Figure 2 Left: positive reaction with the monoclonal anti-p24 antibody in lymph node tissue from an HIV-positive patient during HAART (positive control). Right: negative reaction in bone tissue from an HIV-positive patient with osteonecrosis during HAART and undetectable viral load.

Abbreviation: HAART, highly active antiretroviral therapy.

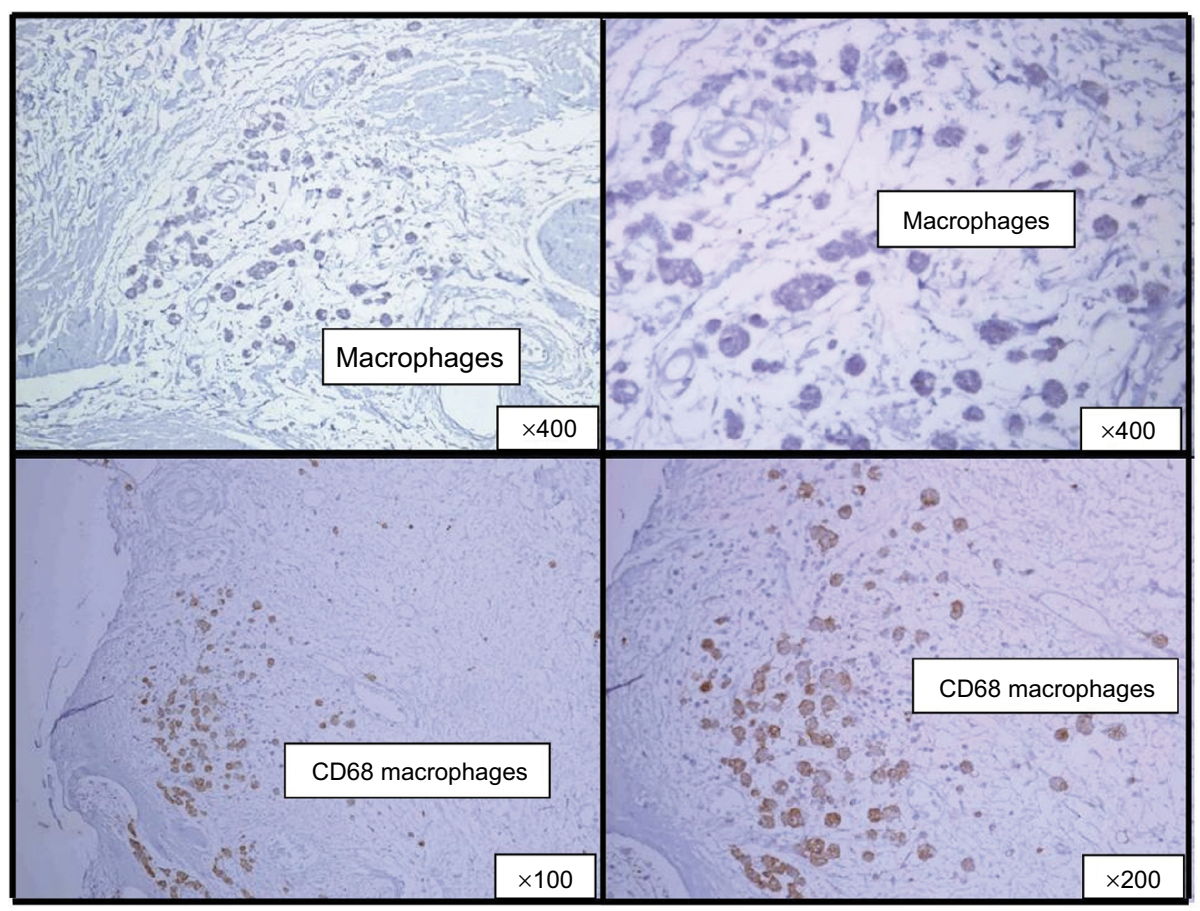

Figure 3 Positive reaction with the monoclonal anti-CD68 antibody in femoral bone tissue of a patient living with HIV.

disorders of fat metabolism contribute, to some extent, as a risk factor for the development of osteonecrosis. In our study, the use of steroids was not identified as a risk factor for the occurrence of this type of bone necrosis, as cited in previous studies. ${ }^{11,14,16}$ In the present study, the majority of patients showed bilateral involvement of the hips with osteonecrosis, which has also been demonstrated previously. ${ }^{17,18}$

In our sample of PLWHIV and osteonecrosis, joint cartilage degeneration was visualized, with the presence of fissures and fibrillations, and this finding was compatible with the diagnosis of osteoarthritis, as well as subchondral sclerosis and subchondral cancellous bone necrosis. The bone matrix showed eosinophilic staining with empty gaps. The bone marrow showed areas of adiponecrosis and granular eosinophilic amorphous material with debris and cell remains. A lymphoplasmocytic inflammatory reaction was observed, with the presence of macrophages containing foamy, vacuolated cytoplasm, as well as the presence of ceroid pigment and occasional granulation tissue. Comparing the HIV-positive and HIV-negative groups, a weaker inflammatory response was detected in the samples of PLWHIV, represented mainly by the presumed macrophages 
visualized by H\&E staining, as well as by large vacuoles formed around areas of necrosis, which were considered as signs of fat cell atrophy. There was no visualization of vessels filled with clots, as described in some cases in the general population, a finding that would support one of the etiological theories of the disease that it is the result of thrombosis of small vessels of the femoral head or embolization of small arteries. ${ }^{19}$ It is also worth mentioning that the presence of ceroid pigment is a marker of tissue aging and was visualized in the majority of the patients living with HIV. This finding gives credence to the theory of early aging in this population.

Immunohistochemical staining was performed in samples of bone tissue from the femoral head to detect the p24 HIV antigen, to confirm the possible macrophages visualized on H\&E staining (CD68), and to verify the large vacuoles observed in conventional histology to rule out the involvement of venous structures through the presence of endothelial (CD31) or lymphatic $\left(\mathrm{D}_{2} 40\right)$ reactions. The p24 antigen test showed negative results in all the 44 patients studied. The aim of the p24 immunohistochemistry assay was to address the potential direct role of HIV in the etiology of osteonecrosis. Obviously, the results obtained do not invalidate this possibility, as implementing techniques to identify etiological agents is difficult in bone tissue due to its nature and the characteristics of preparation of the material for microscopic analysis. In addition to clarifying the role of the virus in the genesis of osteonecrosis, we were also interested in the possibility that the bone tissue acts as a host for the virus, which may contribute to the difficulties associated with eradicating HIV in humans, despite the proven effectiveness of high-power antiretroviral treatment to inhibit key points in the life cycle of the virus.

The limitations of this study include the nonvalidation of the p24 antigen test for bone tissue; however, it is our opinion that using positive and negative control lymph node samples demonstrated the validity of this test. Another limitation was our detection of HIV in necrotized tissue, which may have led to false-negative results. Nevertheless, in the histological slides, not all bones were necrotic, and HIV could have been found in a viable bone. Further research should study the presence of HIV in a nonnecrotic bone.

\section{Conclusion}

The p24 HIV antigen was not detected in the bone tissue of patients living with HIV and osteonecrosis. The anatomopathological findings most frequently observed in the sample of patients living with HIV and osteonecrosis were as follows: extensive necrosis of the bone tissue, large vacuoles filled with fat cells, inflammatory lymphoplasmocytic reaction with macrophages containing foamy vacuolated cytoplasm, and the presence of ceroid pigment.

\section{Author contributions}

All authors contributed toward data analysis, drafting and revising the paper and agree to be accountable for all aspects of the work.

\section{Disclosure}

The authors report no conflicts of interest in this work.

\section{References}

1. Deeks SG, Tracy R, Douek DC. Systemic effects of inflammation on health during chronic HIV infection. Immunity. 2013;39(4): 633-645.

2. Lafferty MK, Fantry L, Bryant J, et al. Elevated suppressor of cytokine signaling-1 (SOCS-1): a mechanism for dysregulated osteoclastogenesis in HIV transgenic rats. Pathog Dis. 2014;71(1):81-89.

3. Martinez E, Jodar Gimeno E, Reyes Garcia R, et al; Grupo de Estudio de Sida; Sociedad Española de Endocrinología y Nutrición; Sociedad Española de Investigación Ósea y del Metabolismo Mineral; Sociedad Española de Fractura Osteoporótica. Documento de consenso: Recomendaciones para el manejo de la enfermedad ósea metabólica en pacientes con virus de la inmunodeficiencia humana [Consensus statement: recommendations for the management of metabolic bone disease in human immunodeficiency virus patients]. Enferm Infecc Microbiol Clin. 2014;32(4):250-258. Spanish [with English abstract].

4. Mondy K, Tebas P. Emerging bone problems in patients infected with human immunodeficiency virus. Clin Infect Dis. 2003;36(Suppl 2):S101-S105

5. Jain RG, Lenhard JM. Select HIV protease inhibitors alter bone and fat metabolism ex vivo. J Biol Chem. 2002;277(22):19247-19250.

6. Parsonage MJ, Wilkins EG, Snowden N, Issa BG, Savage MW. The development of hypophosphataemic osteomalacia with myopathy in two patients with HIV infection receiving tenofovir therapy. HIV Med. 2005;6(5):341-346.

7. Morse CG, Kovacs JA. Metabolic and skeletal complications of HIV infection: the price of success. JAMA. 2006;296(7):844-854.

8. Hardinge K. The direct lateral approach to the hip. J Bone Joint Surg Br. 1982;64(1):17-19.

9. Kirkwood BR, Sterne JAC. Essential Medical Statistics. 2nd ed. Malden: Blackwell Publishing House Science; 2006.

10. Yombi JC, Vandercam B, Wilmes D, Dubuc JE, Vincent A, Docquier PL. Osteonecrosis of the femoral head in patients with type 1 human immunodeficiency virus infection: clinical analysis and review. Clin Rheumatol. 2009;28(7):815-823.

11. Mazzotta E, Agostinone A, Rosso R, et al. Osteonecrosis in human immunodeficiency virus (HIV)-infected patients: a multicentric casecontrol study. J Bone Miner Metab. 2011;29(3):383-388.

12. Barkhordarian A, Ajaj R, Ramchandani MH, et al. Osteoimmunopathology in HIV/AIDS: a translational evidence-based perspective. Pathol Res Int. 2011;2011:359242.

13. Goorney BP, Lacey H, Thurairajasingam S, Brown JD. Avascular necrosis of the hip in a man with HIV infection. Genitourin Med. 1990;66(6):451-452.

14. Fessel WJ, Chau Q, Leong D. Association of osteonecrosis and osteoporosis in HIV-1-infected patients. AIDS. 2011;25(15):1877-1880. 
15. Morse CG, Mican JM, Jones EC, et al. The incidence and natural history of osteonecrosis in HIV-infected adults. Clin Infect Dis. 2007;44(5):739-748.

16. Lawson-Ayayi S, Bonnet F, Bernardin E, et al; Groupe d'Epidémiologie Clinique du SIDA en Aquitaine. Avascular necrosis in HIV-infected patients: a case-control study from the Aquitaine Cohort, 1997-2002, France. Clin Infect Dis. 2005;40(8):1188-1193.
17. Matos MA, Alencar RW, Matos SS. Avascular necrosis of the femoral head in HIV infected patients. Braz J Infect Dis. 2007;11(1):31-34.

18. Habermann B, Eberhardt C, Kurth AA. Total joint replacement in HIV positive patients. J Infect. 2008;57(1):41-46.

19. Kamal D, Alexandru DO, Kamal CK, Streba CT, Grecu D, Mogoanta L. Macroscopic and microscopic findings in avascular necrosis of the femoral head. Rom J Morphol Embryol. 2012;53(3):557-561.

\section{Publish your work in this journal}

HIV/AIDS - Research and Palliative Care is an international, peerreviewed open access journal focusing on advances in research in HIV, its clinical progression and management options including antiviral treatment, palliative care and public healthcare policies to control viral spread. The journal is included in PubMed. The manuscript man- agement system is completely online and includes a very quick and fair
peer-review system, which is all easy to use. Visit http://www.dovepress. $\mathrm{com} /$ testimonials.php to read real quotes from published authors. 